\title{
Validation of a new ovarian malignancy suspicion index for preoperative evaluation of adnexal masses
}

\author{
Shripad Hebbar*, Vijaya Bharathi K.
}

\begin{abstract}
Department of Obstetrics and Gynecology, Kasturba Medical College, Manipal University, Karnataka, India
\end{abstract}
Received: 04 November 2016

Accepted: 30 November 2016

\author{
*Correspondence: \\ Dr. Shripad Hebbar, \\ E-mail: drshripadhebbar@yahoo.co.in
}

Copyright: ( ) the author(s), publisher and licensee Medip Academy. This is an open-access article distributed under the terms of the Creative Commons Attribution Non-Commercial License, which permits unrestricted non-commercial use, distribution, and reproduction in any medium, provided the original work is properly cited.

\begin{abstract}
Background: The currently available ovarian malignancy probability scores incorporate biochemical markers such as CA 125 (Carbohydrate Antigen 125), which is not routinely available in peripheral centers. There is a need for tumour marker independent prediction model to differentiate malignant ovarian masses from their benign counterparts in order to plan appropriate surgery. To formulate and prospectively validate a new Ovarian Malignancy Suspicion Index (OMSI) independent of serum CA 125 level, in preoperative evaluation of adnexal masses admitted for surgery. Methods: This was a combined retrospective and prospective cohort study conducted in a tertiary referral hospital over a period of one and half years. Retrospective sample included 100 subjects who had undergone surgery for adnexal masses and who had definite histopathological report. Detailed data were obtained with respect to age, menopausal status, sonographic findings including solid areas, ascites, mean diameter, bilateralism, and presence of septa. A logistic multivariate regression analysis was carried out to find the best prediction score (OMSI - Ovarian Malignancy Suspicion Index). This model was further evaluated prospectively in 60 subjects for its diagnostic ability to identify benign and malignant ovarian pathology.

Results: OMSI at the cut off value of 3.9 differentiated effectively malignant ovarian mass from benign variety with a good diagnostic performance (Sensitivity 100\%, Specificity 90.5\%, Positive Predictive Value $81.8 \%$ and Negative Predictive Value 100\%) as good as currently recommended RMI (Risk Malignancy Index) score. It was also found that OMSI > 3.9 was associated with positive ultrasound evidence for ovarian malignancy such as presence of thick septae (90\%), solid areas within the tumour (93.8\%), papillary projections (100\%), bilaterality (90\%) and ascites $(100 \%)$.

Conclusions: This study shows that it is possible to derive ovarian malignancy prediction model such as OMSI without including CA 125 with diagnostic ability in par with risk scoring systems such as WHO recommended RMI. Using this model, physicians working in peripheral centers without facilities for estimating serum tumour markers can arrive at the possible diagnosis and plan appropriate management strategies.
\end{abstract}

Keywords: Adnexal mass, CA 125, OMSI, RMI

\section{INTRODUCTION}

Though cervical cancer is still the commonest cancer among women in India, it is not uncommon nowadays to find a good number of ovarian malignancies in tertiary institutions. According to National Cancer Registry Program (NCRP) which is maintained by Indian Council of Medical Research; ovarian cancer contributes to $8 \%$ of all female cancers. ${ }^{1}$ Unfortunately in early stages, the disease is asymptomatic and presents with non-specific symptoms such as dyspepsia, bloating sensation in the stomach, epigastric pain etc.; and these are treated by family physicians. ${ }^{2}$ By the time the disease becomes symptomatic, the stage of the cancer would have already 
been Stage III or IV and even aggressive treatment is associated with treatment failure and recurrence. ${ }^{3}$ Most of these cancers $(>80 \%)$ are epithelial in nature and among epithelial tumours, serous cystadenocarinoma is the commonest variety. ${ }^{4}$ This tumour in particular secretes a tumour antigen known as CA 125 which can be used as a biomarker for screening and follow up of women affected. ${ }^{5}$ Many scoring systems for prediction of ovarian malignancy incorporate CA 125 values in their tumour prediction algorithms, for example; RMI scoring ${ }^{6}$, ROMA index ${ }^{7}, \mathrm{OVA}^{8}$ etc. However, CA 125 estimation is available only in metropolitan cities and hence there is a need for purely demographic and ultrasound based screening method which is as effective as CA 125 based scoring system. The additional advantage of such a screening tool is that it will guide the practicing gynaecologists to refer the case to higher centers with facilities for advanced radical surgery and chemotherapeutic administration whenever required. In the present study, we have derived a new index (Ovarian Malignancy Suspicion Index -OMSI) based on multiple logistic regression analysis conducted on a retrospective cohort and validated the same on new prospective cohort.

\section{METHODS}

This observational study was conducted in the Department of Gynaecology of a tertiary care hospital from December 2015 to June 2016. The study was done in two phases, Phase 1 - index derivation phase, Phase 2 - index validation phase. For the first phase, we studied 100 retrospective cases of women with adnexal masses to derive the index, which was then prospectively applied to 60 women to validate the index. All the patients had ultrasound examination, CA-125 estimation and exploratory laparotomy. The study variables included age, menopause, sonographic findings including solid areas, ascites, mean diameter, bilateralism, and presence of septa. The final diagnosis was based on histopathology of the specimen sent to pathology laboratory. The institutional regulatory body gave permission to conduct the study.

The sample size required for deriving the index was calculated by the assumption given by Harris (1985), who stated that the number of participants should exceed the number of predictors by at least $50 .^{9}$ In our study the numbers of study variables were 8 and we required at least 58 cases to devise the multiple regression formula. However we decided to recruit at least 100 cases to increase the power of the study.

We have used logistic multivariate regression model using SPSS $\mathrm{v} 16$ to estimate weightage for above mentioned study variables. The final composite index (OMSI as shown in Table 4) was subjected to ROC analysis to determine the best cut-off value to differentiate benign from malignant masses and this cutoff was prospectively evaluated in another 60 cases. The corresponding sensitivity, specificity, positive and negative predictive values and accuracy were also calculated.

Table 1: Criteria used to construct OMSI.

\begin{tabular}{|ll|}
\hline Demographic criteria & Ultrasound criteria \\
\hline Age & Mean diameter \\
\hline Menopausal status & Papillary projections \\
\hline & Solid areas \\
\hline & Thick septa \\
\hline & Bilaterality \\
\hline & Ascites \\
\hline
\end{tabular}

\section{RESULTS}

Table 2: Histopathology of ovarian masses in two groups.

\begin{tabular}{|c|c|}
\hline $\begin{array}{l}\text { Index derivation } \\
\text { group }(\mathbf{N}=\mathbf{1 0 0})\end{array}$ & $\begin{array}{l}\text { Index validation group } \\
(\mathrm{N}=60)\end{array}$ \\
\hline $\begin{array}{l}\text { Serous cystadenoma - } \\
13\end{array}$ & Serous cystadenoma - 19 \\
\hline $\begin{array}{l}\text { Mucinous } \\
\text { Cystadenoma - } 20\end{array}$ & Mucinous cystadenoma - 7 \\
\hline Endometrioma - 3 & Endometrioma - 3 \\
\hline Dermoid cyst - 2 & Dermoid cyst - 5 \\
\hline Fibroma ovary - 2 & Paraovarian cyst - 4 \\
\hline $\begin{array}{l}\text { Mature cystic } \\
\text { teratoma - } 10\end{array}$ & Fimbrial cyst - 2 \\
\hline $\begin{array}{l}\text { Serous } \\
\text { Cystadenocarcinoma } \\
-28\end{array}$ & Fibroma ovary - 2 \\
\hline $\begin{array}{l}\text { Mucinous } \\
\text { Cystadenocarcinoma } \\
-15\end{array}$ & Serous cystadenocarcinoma - 5 \\
\hline $\begin{array}{l}\text { Endometrioid } \\
\text { adenocarcinoma - } 3\end{array}$ & $\begin{array}{l}\text { Mucinous cystadenocarcinoma } \\
-6\end{array}$ \\
\hline Dysgerminoma - 2 & $\begin{array}{l}\text { Endometrioid adenocarcinoma } \\
-2\end{array}$ \\
\hline $\begin{array}{l}\text { Clear cell } \\
\text { adenocarcinoma - } 1\end{array}$ & Dysgerminoma - 3 \\
\hline $\begin{array}{l}\text { Immature teratoma - } \\
1\end{array}$ & Granulosa cell tumor -1 \\
\hline
\end{tabular}

Table 2 shows final histopathology of ovarian masses both in OMSI derivation group $(n=100)$ and OMSI validation group $(n=60)$. It is interesting to note that there were both benign and malignant cases in both the groups, implicating that histopathology is the final gold standard in evaluation of adnexal masses.

Table 3 shows the regression weightage for predictor variables. It is interesting to note that menopausal status $(\mathrm{R}=0.851)$, septal thickness $>3 \mathrm{~mm}(\mathrm{R}=0.776)$, presence of ascites $(\mathrm{R}=0.756)$, followed by solid areas within the tumour $(\mathrm{R}=0.686)$ had high coefficients. All the regression coefficients were incorporated into the formula to derive the predictor model. 
Table 4 shows the new derived model (OMSI - Ovarian Malignancy Suspicion Index) derived from the findings subjects recruited in formula derivation group $(\mathrm{N}=100)$.

Table 3: Multiple logistic regression analysis to find the weightage for each variable.

\begin{tabular}{|llll|}
\hline Variable & $\begin{array}{l}\text { Regression } \\
\text { Coefficient }\end{array}$ & $\begin{array}{l}\text { T } \\
\text { value }\end{array}$ & Significance \\
\hline Age & 0.036 & 13.753 & $<0.001$ \\
\hline Menopause & 0.851 & 15.113 & $<0.001$ \\
\hline $\begin{array}{l}\text { Mean } \\
\text { diameter }\end{array}$ & 0.168 & 8.24 & $<0.001$ \\
\hline Septa & 0.776 & 11.389 & $<0.001$ \\
\hline Solid areas & 0.686 & 8.173 & $<0.001$ \\
\hline Papillary & 0.599 & 5.996 & $<0.001$ \\
\hline Bilaterality & 0.572 & 4.921 & $<0.001$ \\
\hline Ascites & 0.756 & 9.377 & $<0.001$ \\
\hline
\end{tabular}

This model was applied to all the 100 patients in the index derivation group and the values were calculated. The values ranged from 2.17 to 8.34 . ROC analysis was carried out to determine which cut off identifies the malignant cases and it was found that OMSI value of 3.9 differentiated benign from malignant ovarian tumours (Area under the curve $0.89, \mathrm{p}<0.001$ ). The ROC graph is shown in Figure 1.

OMSI cut off value of 3.9 was prospectively evaluated in next 60 patients. Table 5 shows descriptive statistics for OMSI. It was found that in 22 patients who had OMSI>3.9, the parameters such as age of the patient, mean tumour diameter and CA 125 levels were significantly more compared to those 38 patients who had OMSI values less than 3.9.

\section{Table 4: The New Ovarian Malignancy Suspicion Index - OMSI.}

The New Ovarian Malignancy Suspicion Index - OMSI OMSI $=0.036($ Age $)+0.851$ (if menopausal $)+$ 0.168 (Mean Diameter in CM) +0.776 (if Septal thickness $>3 \mathrm{MM})+0.686$ (if tumour is solid) +0.599 (if papillary projections present $)+0.572$ (if bilateral) +0.756 (if ascites present).

Table 5: Descriptive statistics for age, diameter and CA 125 levels according to OMSI cut-offs.

\begin{tabular}{|llllll|}
\hline & OMSI $<3.9, \mathrm{n}=38$ & \multicolumn{2}{c|}{ OMSI $>3.9, \mathrm{n}=22$} & Statistics \\
\hline Parameter & Mean & SD & Mean & SD & P- Value \\
\hline Age $(\mathrm{yrs})$ & 41 & 4.9 & 58 & 9.2 & $<0.001$ \\
\hline Mean diameter $(\mathrm{cm})$ & 7.2 & 1.4 & 9.2 & 2.3 & $<0.001$ \\
\hline CA $125(\mathrm{u} / \mathrm{mL})$ & 21 & 25 & 892 & 1714 & $<0.001$ \\
\hline
\end{tabular}

Table 6: Association between risk factors for ovarian malignancy and OMSI cut-off.

\begin{tabular}{|lll|}
\hline Risk factor for malignancy & $<3.9$ & $>3.9$ \\
\hline Menopause & $0(0)$ & $17(100)$ \\
\hline Thick septa & $1(10)$ & $9(90)$ \\
\hline Solid areas & $1(6.2)$ & $16(93.8)$ \\
\hline Papillary projections & $0(0)$ & $8(100)$ \\
\hline Ascites & $0(0)$ & $15(100)$ \\
\hline Bilaterality & $1(10)$ & $9(90)$ \\
\hline *Some cases had multiple findings & \\
\hline
\end{tabular}

Further in this validation group of 60 patients, risk factors for ovarian cancer such as menopausal status, ultrasound evidence of thick septae, solid areas within the tumour, papillary projections, ascites and bilaterality were studied. Table 6 indicates that whenever OMSI was $>3.9$, all these risk factors accounted for maximum incidence. Finally histopathological analysis of resected tumours indicated presence of malignancy in 18 patients and all these subjects had OMSI values above 3.9.

Finally the efficacy of derived OMSI parameter with respect to sensitivity, specificity, positive predictive value, negative predictive value and accuracy was compared with RMI scoring system, single ultrasound parameters such as thick septa, papillary projections, involvement of both the ovaries and presence of ascites as shown in Table 7. It can be seen that OMSI had small edge over of RMI, but definitely performed well compared to single ultrasound parameters.

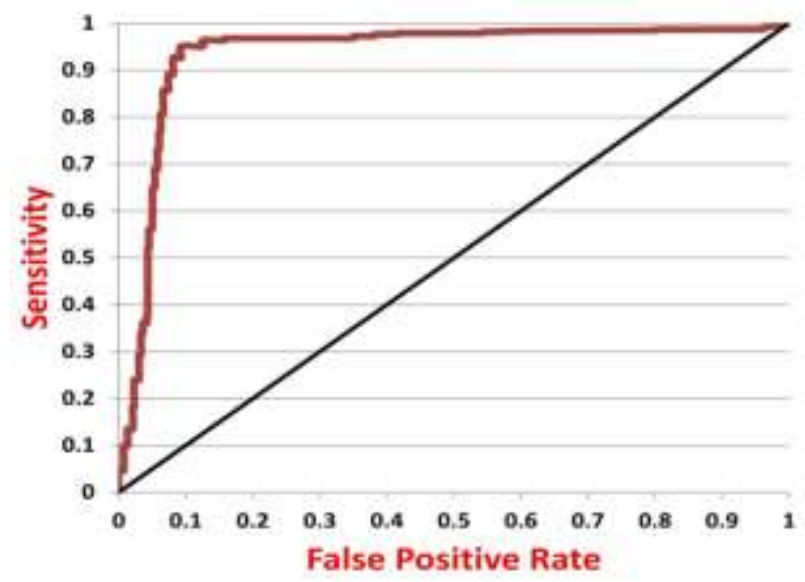

Figure 1: ROC curve for OMSI for prediction of ovarian malignancy. 
Table 7: Overall efficacy of different parameters in prediction of ovarian malignancy.

\begin{tabular}{|c|c|c|c|c|c|c|c|c|c|}
\hline Parameters & TP & $\mathrm{TN}$ & FP & FN & $\begin{array}{l}\text { Sensitivity } \\
(\%)\end{array}$ & $\begin{array}{l}\text { Specificity } \\
(\%)\end{array}$ & $\begin{array}{l}\text { PPV } \\
(\%)\end{array}$ & $\begin{array}{l}\text { NPV } \\
(\%)\end{array}$ & Accuracy \\
\hline OMSI $>3.9$ & 18 & 38 & 4 & 0 & 100.0 & 90.5 & 81.8 & 100.0 & 93.3 \\
\hline RMI1 >200 & 18 & 38 & 3 & 1 & 94.7 & 92.7 & 85.7 & 97.4 & 93.3 \\
\hline Septa $>3 \mathrm{~mm}$ & 8 & 40 & 2 & 10 & 44.4 & 95.2 & 80.0 & 80.0 & 80.0 \\
\hline $\begin{array}{l}\text { Papillary } \\
\text { projections }\end{array}$ & 8 & 42 & 0 & 10 & 44.4 & 100.0 & 100.0 & 80.8 & 83.3 \\
\hline Bilaterality & 7 & 39 & 3 & 11 & 38.9 & 92.9 & 70.0 & 78.0 & 76.7 \\
\hline Ascites & 14 & 41 & 1 & 4 & 77.8 & 97.6 & 93.3 & 91.1 & 91.7 \\
\hline
\end{tabular}

\section{DISCUSSION}

The presurgical workup for ovarian tumour is a major task for a gynaecological surgeon. An element of suspicion towards the malignant aetiology changes the plan of surgery, and extent of surgery. For example, a benign ovarian cyst can be tackled by laparoscopy, cyst removal may be facilitated through the aspiration of cyst fluid and accidental rupture of cyst during the procedure does not harm the patient anyway. The surgery can also be done through pfannenstiel incision, a preferred surgical incision for the women for cosmetic reason, as there is no need for extensive pelvic dissection. On the contrary, if the mass is of malignant nature, the route is always laparotomy, which involves a liberal midline incision which can be extended into the upper abdomen and a complex procedure such as pelvic and para-aortic lymphadenectomy, total or partial omentectomy in addition to total abdominal hysterectomy with removal of bilateral adnexa. Hence a proper preoperative evaluation will differentiate between benign and malignant ovarian neoplasm and facilitate the transfer the patient to a tertiary care center where multimodal treatment for ovarian cancer is available comprising of oncosurgeon and medical oncologist.

Abdominal and pelvic ultrasounds have contributed significantly to characterise the pelvic masses. Additional studies such 3D ultrasound, estimation of tumour vascularity by Doppler pulsatility index further adds to accuracy of imaging modality. However, in the presence of clinical signs such as bilaterality, fixity, ascites etc., nothing much is gained through the ultrasound studies. Researchers in the past have tried to improve the diagnostic ability of ultrasound by adding preoperative levels of CA 125, which is a glycoprotein secreted by epithelial ovarian tumors in their scoring systems. It was Jacob et al, in 1990, who first described an index called Risk of Malignancy Index (RMI) by adopting a simplified multiplication model from the product of CA 125 in U/mL, menopausal status and ultrasound findings. ${ }^{10}$

RMI is the product of: CA $125 \mathrm{X}$ Menopausal score X Sonographic score. The value for CA 125 is its absolute level (Units per mL). Menopausal score depends upon the menopausal status, if patient is still menstruating she will get the score of 1 and if has achieved menopause then the score will be 3 . The ultrasound score depends upon five sonographic features; multilocularity, mural solid components, bilaterality, presence of ascitic fluid and evidence of metastatic spread. When no findings are present, then the score is zero, the score is one for presence of one finding and if more than one finding are present, score is 3 irrespective of numbers of ominous findings (meaning the maximum score is 3 whether 2 or 3 or 4 or all 5 findings are present). This model is associated with inherent deficiencies. For example; elevated levels have been found in several benign conditions such as endometriosis, abdominal tuberculosis and ovarian tumours other than serous cystadenocarinoma may not secrete this glycoprotein.

In our study, only 28 cases in OMSI derivation group and 5 cases in OMSI validation group belonged to serous epithelial malignant tumours which exhibited significant rise in serous CA 125 levels. Similarly 15 cases in validation group and 6 cases in derivation group belonged to mucinous carcinomas but not all of them had raised CA 19-9 levels. Thus it is important to remember that these carbohydrate oncoproteins though raised in respective tumours, will not represent other cancerous conditions of the ovary and hence will not serve as universal markers and moreover CA 125 estimation is not available in smaller peripheral centers, blood has to be sent to sophisticated referral laboratory by courier and report takes significant time there by delaying the management. Hence there is a need for prediction model independent of CA 125, which has comparable diagnostic accuracy to other scoring models incorporating this tumour marker.

Similarly only the menopausal state of the women is considered to contribute one or three marks. For example if there are two menopausal patients aged 45 and 65 years are given same weightage, whereas two other patients let us say, 46 years, one is premenopausal and the other is postmenopausal are scored 1 and 3 respectively and hence the second one will have three times the score, if other two parameters are scored similarly. This tells us 
the importance of including the absolute age in addition to menopausal status in the formulation of predictive model.

Now coming to the ultrasound parameters, if ultrasound shows benign features, then the score is zero and the final RMI is also zero irrespective of high scores given for age and CA 125 levels. And even more there is no weightage given for size of the cyst. Even the maximum score is 3 in spite all positive ultrasound findings.

To address these issues, there are three modifications of RMI (the original one described by Jacob et al is considered as RMI $1^{10}$, the others in series are called as RMI $2^{11}$, RMI $3^{12}$ and RMI4 ${ }^{13}$ ). However even with these risks scoring system, not all issues are resolved well, not easily remembered and one has to look up at their tables to calculate the scores. Hence there is a need for simplified formula which can address all these issues.

An Iranian group formulated a prediction model comprising of only five variables (Age, Tumour size in $\mathrm{cm}$, presence of solid areas, ascites and bilateralism) and could achieve sensitivity of $78 \%$ and specificity of $73 \%$ without including CA 125 levels. $^{14}$ The increased accuracy in our model is due to inclusion of age of patient, mean tumour diameter, ultrasound findings of thick septa and papillary projections. This can be further improved by adding several other parameters such as Body Mass Index (BMI), duration of OC pill intake in the past, parity (especially number of full term deliveries), Hormone Replacement Therapy and previous unilateral oophorectomy at the time of hysterectomy.

A largest study in this aspect is EPIC (European Prospective Investigation into Cancer and Nutrition study), which examined risk factors in 2,02,206 women from European countries. ${ }^{15}$ It was found that menopause at older age, prolonged hormone replacement therapy and obesity increased ovarian cancer risk. Prior surgical extirpation of one of the ovaries, prior use of estrogen progesterone contraceptive pills and multiparity were found to be protective against development of ovarian cancer. They formulated a prediction model (which had 12 variables) had the discriminatory power (overall concordance index) of 0.64 (95\% confidence interval (CI): $0.57,0.70)$. However the computed model is too complex, difficult to understand and not easy to calculate the values in routine practice.

Our risk model is simple, more accurate and can be calculated with simple calculator programs available in all computers and mobile devices. We have already described the methodology which can be applied to a larger set of patients and coefficients can further be modified to further increase the accuracy of ovarian malignancy prediction models.

\section{CONCLUSION}

This study proves that it is possible to formulate risk scoring method using various demographic factors and ultrasound findings independent of CA 125 estimation. This prediction model has comparable diagnostic accuracy to other scoring models incorporating tumour biomarker. Preoperative estimation of ovarian malignancy suspicion index will aid in in-depth metastatic workup, preparation of the patient for more radical surgery, change of plan to neoadjuvant chemotherapy, referral to surgical oncology unit and moreover counseling the patient to adverse disease outcome.

\section{Funding: No funding sources}

Conflict of interest: None declared

Ethical approval: The study was approved by the Institutional Ethics Committee

\section{REFERENCES}

1. National Cancer Registry Programme. Available from:

http://ncrpindia.org/ALL_NCRP_REPORTS/PBCR_ REPORT_2012_2014/index.htm. Accessed November 6, 2016.

2. Bankhead CR, Collins C, Stokes-lampard H, et al. Identifying symptoms of ovarian cancer: a qualitative and quantitative study. BJOG. 2008;115(8):1008-14.

3. Jelovac D, Armstrong DK. Recent progress in the diagnosis and treatment of ovarian cancer. CA Cancer J Clin. 2011;61(3):183-203.

4. Cho KR, Shih IeM. Ovarian cancer. Annu Rev Pathol. 2009;4:287-313.

5. Scholler N, Urban N. CA125 in ovarian cancer. Biomark Med. 2007;1(4):513-23.

6. Aktürk E, Karaca RE, Alanbay I, et al. Comparison of four malignancy risk indices in the detection of malignant ovarian masses. J Gynecol Oncol. 2011;22(3):177-82.

7. Dikmen ZG, Colak A, Dogan P, Tuncer S, Akbiyik F. Diagnostic performances of CA125, HE4, and ROMA index in ovarian cancer. Eur J Gynaecol Oncol. 2015;36(4):457-62.

8. Grenache DG, Heichman KA, Werner TL, Vucetic Z. Clinical performance of two multi-marker blood tests for predicting malignancy in women with an adnexal mass. Clin Chim Acta. 2015;438:358-63.

9. Harris RJ. A primer of multivariate statistics (2nd Ed.). New York: Academic Press. 1985.

10. Jacobs I, Oram D, Fairbanks J, Turner J, Frost C, Grudzinskas JG. A risk of malignancy index incorporating CA 125, ultrasound and menopausal status for the accurate preoperative diagnosis of ovarian cancer. $\mathrm{Br} \mathrm{J}$ Obstet Gynaecol. 1990;97(10):922-9.

11. Tingulstad S, Hagen B, Skjeldestad FE, et al. Evaluation of a risk of malignancy index based on serum CA125, ultrasound findings and menopausal 
status in the pre-operative diagnosis of pelvic masses. Br J Obstet Gynaecol. 1996;103(8):826-31.

12. Tingulstad S, Hagen B, Skjeldestad FE, Halvorsen T, Nustad K, Onsrud M. The risk-of-malignancy index to evaluate potential ovarian cancers in local hospitals. Obstet Gynecol. 1999;93(3):448-52.

13. Yamamoto Y, Yamada R, Oguri H, Maeda N, Fukaya T. Comparison of four malignancy risk indices in the preoperative evaluation of patients with pelvic masses. Eur J Obstet Gynecol, 2009;144:163-7.
14. Arab M, Yaseri M, Farzaneh M. The construction and validation of a new ovarian malignancy probability score (OMPS) for prediction of ovarian malignancy. Iranian J Cancer Prev. 2012;3:132-38.

15. Li K, Hüsing A, Fortner RT. An epidemiologic risk prediction model for ovarian cancer in Europe: the EPIC study. Br J Cancer. 2015;112(7):1257-65.

Cite this article as: Hebbar S, Vijaya Bharathi K. Validation of a new ovarian malignancy suspicion index for preoperative evaluation of adnexal masses. Int J Reprod Contracept Obstet Gynecol 2017;6:2405 . 